\title{
两种形态分析方法对冰川退缩时间序列土壤中 磷的生物有效性评价
}

吴艳宏 ${ }^{(1 *}$, Jörg PRIETZEL ${ }^{(2)}$, 周俊 ${ }^{(1)}$ ，邴海健 ${ }^{(1)}$ ，罗辑 ${ }^{(1)}$, 余东 ${ }^{(1)}$, 孙守琴 ${ }^{(1)}$,

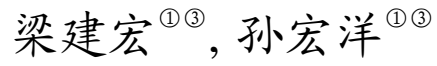

(1) 中国科学院水利部成都山地灾害与环境研究所, 中国科学院山地表生过程和生态调控重点实验室, 成都 610041;

(2) Technische Universität München, Lehrstuhl für Bodenkunde, Munich 85354, Germany;

(3) 中国科学院大学地球科学学院, 北京 100049

*E-mail: yhwu@imde.ac.cn

收稿日期: 2013-07-29; 接受日期: 2013-12-04; 网络版发表日期: 2014-08-05

国家自然科学基金项目(批准号: 41272200)、中国科学院重要方向性项目(编号: KZCX2-YW-BR-21)和中国科学院创新团队国际合作伙伴 计划(编号: KZZD-EW-TZ-06)资助

摘要士壤磷的生物有效性是陆地生态系统安全和初始生产力的重要影响因素.土壤中 磷的生物有效性由其形态组成决定. 常用的磷形态分类方法是 Hedley 提出的连续提取法, 即通过不同化学强度的提取剂对土壤样品进行分步提取, 各级提取剂提取出的磷被定义为 不同形态的磷. 近年来又推出了 X 射线吸收近边结构法(XANES), 利用在分子水平上给出 目标元素周围的局部结构和化学信息的优势, 能区分出与 $\mathrm{Fe}, \mathrm{Al}$ 和 $\mathrm{Ca}$ 等金属结合的磷的 形态. 本文利用改进的 Hedley 连续提取法和 XANES 方法对贡嘎山海螺沟冰川退缩迹地上 土壤中磷的形态进行了分析, 在比较两种方法优缺点的基础上, 获得了海螺沟冰川退缩 120 年序列上土壤磷的生物有效性的变化. 结果表明: Hedley 连续提取法对即时生物可利 用磷能有准确的判识 (Resin-P 和 $\left.\mathrm{NaCHO}_{3}-\mathrm{P}\right)$, 而 XANES 方法对金属结合态的磷有很好的 判识. 海螺沟冰川退缩时间序列土壤中钻铝结合态的磷主要为原生矿物磷, 随成土作用增 强而减少。即时生物可利用磷从 30 年后迅速增加, 促使了植被形成和演替.

关键词

土壤

磷形态

Hedley 连续提取

XANES

海螺沟
磷 $(\mathrm{P})$ 是重要的生命元素. 在陆地生态系统中, 磷往往与氮一道成为影响生态系统安全、决定初始生 产力的重要限制因素(Vitousek 等, 2010). 此外土壤 中的磷等元素可能是气候因素之外决定植被带谱和 林线形成的重要因素(Sala 等, 2000; Ducić 等, 2011), 而对于因冰川退缩形成的土壤序列上的原生植被演
替，土壤中的磷也起到关键作用(Chapin 等, 1994; Wu 等, 2013).

土壤中磷以无机(矿物)和有机(有机质)两种形式 存在, 从形态上又分为溶解态和颗粒吸附态. 溶解态 的磷为正磷酸盐, 在自然界中仅占很小的比例, 颗粒 态的磷吸附于铝 $(\mathrm{Al})$ 、铁 $(\mathrm{Fe})$ 等金属氧化物或氢氧化

中文引用格式: 吴艳宏, Prietzel J, 周俊, 等. 2014. 两种形态分析方法对冰川退缩时间序列土壤中磷的生物有效性评价. 中国科学: 地球科学, 44: 2006-2015 英文引用格式: Wu Y H, Prietzel J, Zhou J, et al. 2014. Soil phosphorus bioavailability assessed by XANES and Hedley sequential fractionation technique in a glacier foreland chronosequence in Gongga Mountain, Southwestern China. Science China: Earth Sciences, 57: 1860-1868, doi: $10.1007 / \mathrm{s} 11430-013-4741-\mathrm{z}$ 
物以及粘土矿物的表面, 生物有效磷是指生物可以 直接吸收利用的磷, 其形态主要为溶解态正磷酸盐, 其余形态不能直接为植物利用或完全不能被植物吸 收利用(被称之为闭蓄态磷)(Walker 和 Syers, 1976; Gonsiorczyk 等, 1998; Reynolds 和 Davies, 2001). 因 此, 对土壤中磷的形态研究, 获取土壤中磷的生物有 效性信息, 是探讨磷对陆地生态系统影响、建立磷与 植被带谱形成及植被演替关系的重要基础.

对土壤磷形态的分析多采用连续提取的方法, 即采用不同类型的选择性提取剂连续地对土壤样品 进行提取, 各级提取剂提出的磷被定义为不同形态 的磷. 目前较成熟的连续提取方法由 Hedley等(1982) 提出并被不断改进(Cross 和 Schlesinge, 1995; Cassagne 等, 2000, Zhou 等, 2013), 根据提取剂的不同分 为树脂磷 $(\operatorname{Resin}-\mathrm{P})$ 、碳酸氢钠磷 $\left(\mathrm{NaHCO}_{3}-\mathrm{P}\right)$ 、氢氧化 钠磷 $(\mathrm{NaOH}-\mathrm{P})$ 和盐酸磷 $(\mathrm{HCl}-\mathrm{P})$ 等. 然而, 这种连续 提取区分出的磷的形态并不能明确地对应于磷的化 学形态, 不能准确区分出铁结合态、铝结合态还是钙 结合态(Hunger 等, 2005; Kar 等, 2011), 这对准确评 价磷的生物有效性带来困难. 近年来, 逐渐发展起了 多种技术手段来弥补这一缺陷, 如基于同步辐射光 源的 $\mathrm{X}$ 射线吸收近边结构法(XANES), 在分子水平 上给出目标元素周围的局部结构和化学信息 (Hesterberg 等, 1999; Beauchemin 等, 2003; Ravel 和 Newville, 2005; Lombi 等, 2006; 刘瑾等, 2011; Prietzel 等, 2013), 从而准确区分与不同金属氧化物 相结合的磷. 然而, 迄今为止只有两项研究工作, 比 较了 Hedley 连续提取和 XANES 方法的磷形态数据, 认为 XANES 方法直接区分出了土壤中无机磷的形态, 这是 Hedley 连续提取法所做不到的(Beauchemin 等, 2003; Kruse 和 Leinweber, 2008). 但也有研究注意到 一些吸附于矿物或有机质的磷酸盐有着相似的能谱 (Peak 等, 2002), 给判断磷形态带来较大误差(Ajiboye 等, 2008). 因此一些研究工作将两种方法同时应用, 以获得对土壤磷形态组成的更好把握 (Kruse 和 Leinweber, 2008).

本研究将通过贡嘎山海螺沟冰川退缩迹地上 120 年的土壤序列, 利用改进的 Hedley 连续提取和 XANES 两种方法对土壤磷形态进行分析, 准确获取 土壤中磷形态组成随时间和土壤发育变化的特征, 为冰川退缩区土壤发育和植被原生演替研究提供数 据. 同时比较两种方法在土壤磷形态研究中的优缺
点, 为完善土壤中磷的生物地球化学循环研究提供 新途径.

\section{1 研究区概况}

贡嘎山属于横断山系, 位于青藏高原东缘, 是四 川盆地与青藏高原的过渡地带, 地理范围介于 $101^{\circ} 30^{\prime} \sim 102^{\circ} 15^{\prime} \mathrm{E}$ 和 $29^{\circ} 20^{\prime} \sim 30^{\circ} 20^{\prime} \mathrm{N}$, 面积约 $1 \times 10^{4}$ $\mathrm{km}^{2}$, 主峰海拔 $7556 \mathrm{~m}$, 其高度居青藏高原东缘山峰 之首. 区内地质构造异常复杂, 新构造运动强烈, 地 貌上为高山峡谷类型, 环绕贡嘎山山巅四周, 发育数 十条亚洲最大的现代海洋性冰川. 小冰期以来, 随着 全球气温的不断上升, 各地冰川不断退缩, 如贡嘎山 地区的海螺沟冰川退缩明显, 留下多道终碛与侧碛, 在距冰川末端水平距离 $2 \mathrm{~km}$ 左右, 垂直高差 100 多 米的冰川退缩迹地上, 土壤和植被逐渐发育, 经历了 裸地-草本地被、柳-沙棘-水冬瓜、水冬瓜、云冷杉桦-杜鹃和云冷杉等五个阶段的植被原生演替, 并形 成较为清晰的植被带谱(李逊和熊尚发, 1995; 钟祥 浩等, 1999; Li 等, 2010)(图 1).

\section{2 样品采集和分析方法}

采样时间为 2010 年 9 月. 按照土壤年龄(钟祥浩 等, 1999) 由小到大的顺序采样, 共设置 7 个年龄梯度 $(0,12,30,40,52,80$ 和 120 年), 每个样点布置 3 个剖 面. 由于土壤发育时间较短, 所有的土壤剖面都未发 现 B 层，因此，按照以下层次采集土样：A层，暗棕色， 主要为腐殖质土; $\mathrm{C}$ 层, 棕灰色, 砂粒, 母质层. 年龄 为 0 年和 12 年的样点, 尚未有土壤发育, 采集 $0 \sim 10$ $\mathrm{cm}$ 的细粒物质. 每个样品采集约 $1 \mathrm{~kg}$ 土壤, 用干净 的聚氯乙烯塑料袋保存, 带回实验室后用烘箱烘干 $\left(45^{\circ} \mathrm{C}\right)$, 烘干后的土样过 $2 \mathrm{~mm}$ 篎.

土样的 $\mathrm{pH}$ 采用玻璃电极法测定(水土比例 $1: 2.5$ ). 总氮(TN)含量采用元素分析仪测定. 总磷(TP)采用硝 酸-氢氟酸-高氯酸湿法消解; 无定形铁 ( Feo ) 和无定 形铝(Alo) 采用酸性草酸铵溶液提取 $\left(25^{\circ} \mathrm{C}\right)$; 提取后 的 TP, Feo 和 Alo 采用美国 LEEMAN LABS ROFILE 电感耦合等离子体原子发射光谱仪(ICP-AES)测定, 标准为美国 $\mathrm{SPEX}^{\mathrm{TM}}$ 标准溶液.

采用改进后的 Hedley 法(Tiessen 和 Moir，1993) 提取不同形态的土壤 P. 取烘干过篮后的土壤样品 


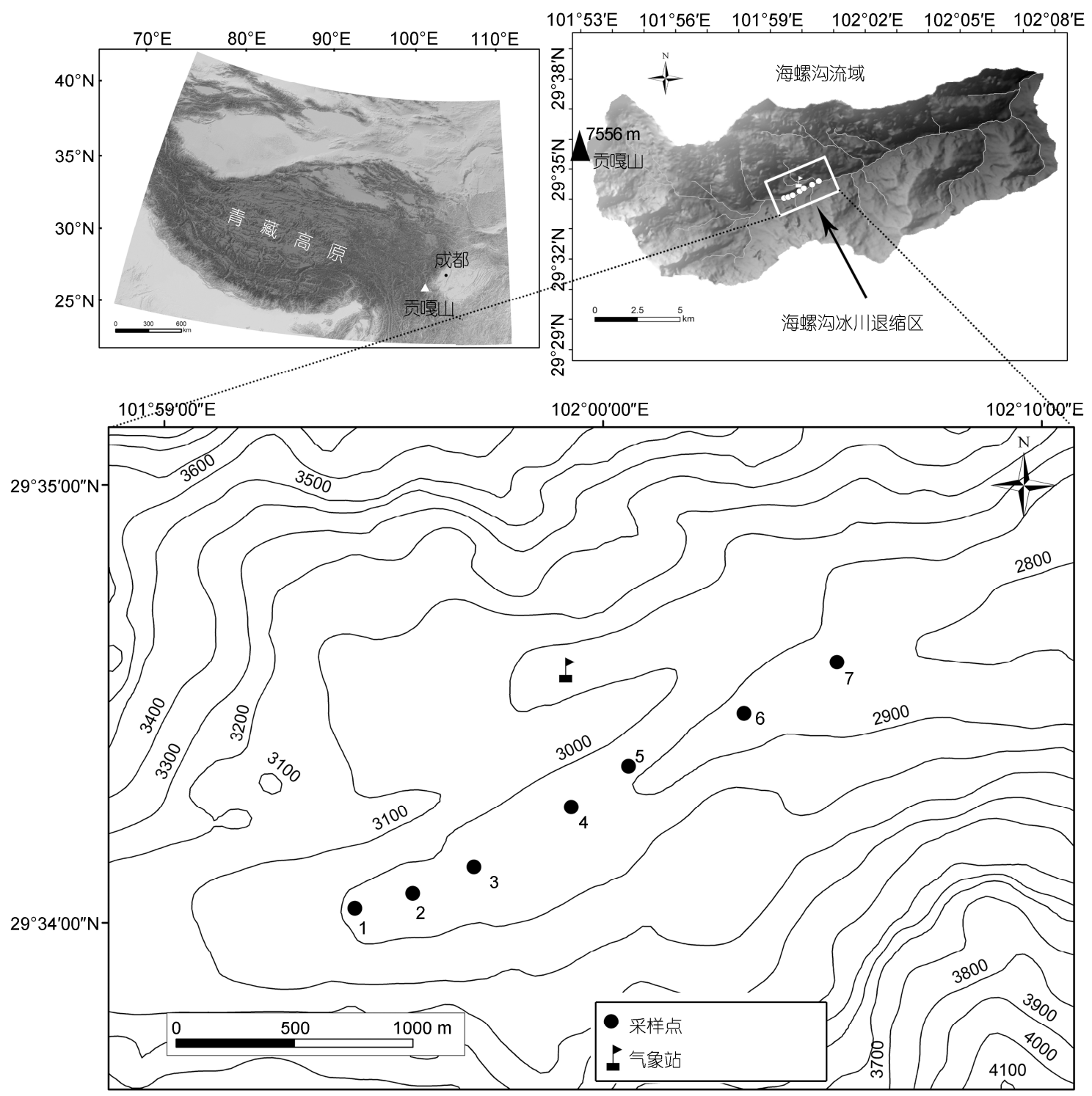

图 1 采样位置图

$0.5 \mathrm{~g}$ (2 个重复), 置于 $50 \mathrm{~mL}$ 干净的聚氯乙烯离心管 中, 每种形态 $\mathrm{P}$ 的化学提取剂如下. Resin-P: 2 片 $\mathrm{HCO}^{3-}$ 饱和的 $\mathrm{AEM}\left(\right.$ 阴离子交换树脂膜, $\mathrm{BDH}^{\circledR}$, $551642 \mathrm{~S}, 9 \mathrm{~mm} \times 62 \mathrm{~mm}), 20 \mathrm{~mL}$ 去离子水; $\mathrm{NaHCO}_{3}-\mathrm{P}$ : $30 \mathrm{~mL} 0.5 \mathrm{M}$ 的 $\mathrm{NaHCO}_{3}$ 溶液; NaOH-P: $30 \mathrm{~mL} 0.1 \mathrm{M}$ 的 $\mathrm{NaOH}$ 溶液; DHCl-Pi: $30 \mathrm{~mL} 1 \mathrm{M}$ 的 $\mathrm{HCl}$ 溶液; CHCl-P: $10 \mathrm{~mL}$ 浓盐酸, $80^{\circ} \mathrm{C}$ 的水浴锅中消煮 $10 \mathrm{~min}$. 除 CHCl-P 外, 其余形态的 $\mathrm{P}$ 在提取过程中均需振荡 $16 \mathrm{~h}\left(25^{\circ} \mathrm{C}\right.$, 往复振荡 $) . \mathrm{NaHCO}_{3}-\mathrm{P}, \mathrm{NaOH}-\mathrm{P}$ 和 $\mathrm{CHCl}-$ $\mathrm{P}$ 提取液分为两份, 一份直接测定磷酸根离子的浓度, 分别为 $\mathrm{NaHCO}_{3}-\mathrm{Pi}, \mathrm{NaOH}-\mathrm{Pi}$ 和 CHCl-Pi; 另一份经
高压灭菌器蒸煮后 $\left(121^{\circ} \mathrm{C}, 2 \mathrm{~h}\right)$ 测定其中的磷酸根 离子浓度, 其结果与未消煮提取液的浓度之差, 为对 应形态的有机磷的浓度, 分别为: $\mathrm{NaHCO}_{3}-\mathrm{Po}$, $\mathrm{NaOH}-\mathrm{Po}$ 和 CHCl-Po. 磷酸根的测定基于钼蓝比色 法, 采用 SHIMADU UV1750 进行(波长 $700 \mathrm{~nm}$ ). 残 余态磷为 ICP-AES 测得的 TP 浓度与上述各形态 P 浓 度之差.

XANES 法: 详细方法见 Prietzel 等(2013). 采用 Aldrich 公司的 6 个不同形态的磷标准物质来区分磷 形态, 同步辐射源由泰国 Synchrotron Light Research Institute(SLRI)提供, 分析测定在 SLRI 完成. 


\section{3 结果}

\section{1 土壤性质}

海螺沟冰川退缩区土壤发育处于早期阶段，土 壤理化性质与土壤发育时间密切相关( $\mathrm{He}$ 和 Tang, 2008)，土壤层较薄，早期阶段分层不明显. 0 年和 12 年样点处, 为土壤发育的最开始阶段, 缺乏 $\mathrm{A}$ 层, 因 此在图 2 中 0 年和 12 年处土壤性质数据用于比较其 他时期土壤 $\mathrm{A}, \mathrm{C}$ 层数据. $\mathrm{pH}$ 在 0 和 12 年处均为 7.3, 随时间逐渐下降，最低值出现于 80 年处的 $\mathrm{A}$ 层(3.6), $\mathrm{C}$ 层 $\mathrm{pH}$ 略高于 $\mathrm{A}$ 层. 总磷含量一直呈减少趋势, 前 52 年 $\mathrm{A}, \mathrm{C}$ 层差异不明显, 而随着土壤发育, 在 80 年 和 120 年处 $\mathrm{A}$ 层总磷明显低于 $\mathrm{C}$ 层. 与总磷含量趋 势相反, 有机碳和总氮在 $\mathrm{A}$ 层中随土壤发育而增加, 在 40 年处分别上升到 165.6 和 $12.5 \mathrm{~g} \mathrm{~kg}^{-1}$ 后，维持在 较高含量, 而 $\mathrm{C}$ 层中有机碳和总氮较低, 基本没有太 大变化. 无定形铁、铝含量随时间增加, 在 $\mathrm{A}$ 层的增 加更为明显，而在 $\mathrm{C}$ 层 40 年后略有下降.

3.2 连续提取方法所获得的海螺沟冰川退缩区土 壤磷形态特征

C 层土壤中几乎只有 DHCl-P, CHCl-P 和 $\mathrm{NaOH}-\mathrm{P}$ 三种形态，尤其是 DHCl-P 其占到总磷的 92\% 97\%, CHCl-P占 3\% 5\%, NaOH-P占 1\% 3\%, 而 Resin-P和 $\mathrm{NaCHO}_{3}-\mathrm{P}$ 两种形态几乎可以忽略(图 3). C 层有机磷含量很低, 只占总磷的 $1 \%$ ～3\%, 主要为

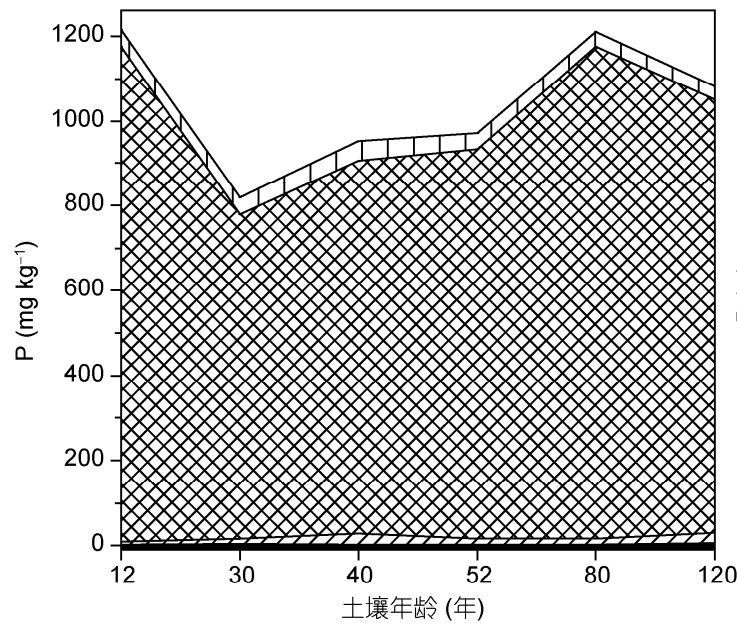

$\mathrm{NaOH}$ 提取态有机磷.

A 层中土壤磷形态组成发生了很大变化, 最明 显的特征是 DHCl-P 的持续减少和其他形态磷含量的 增加. 12 年处缺失 $\mathrm{A}$ 层, 以 $\mathrm{C}$ 层磷形态特征与其他时
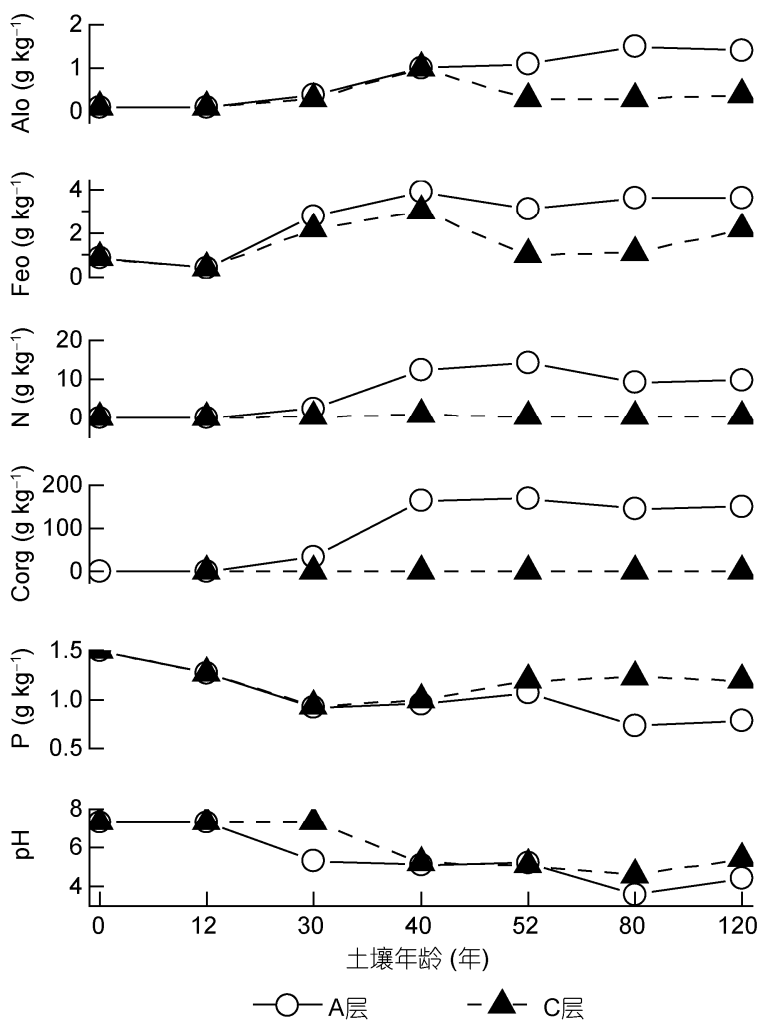

图 2 土壤性质随时间变化

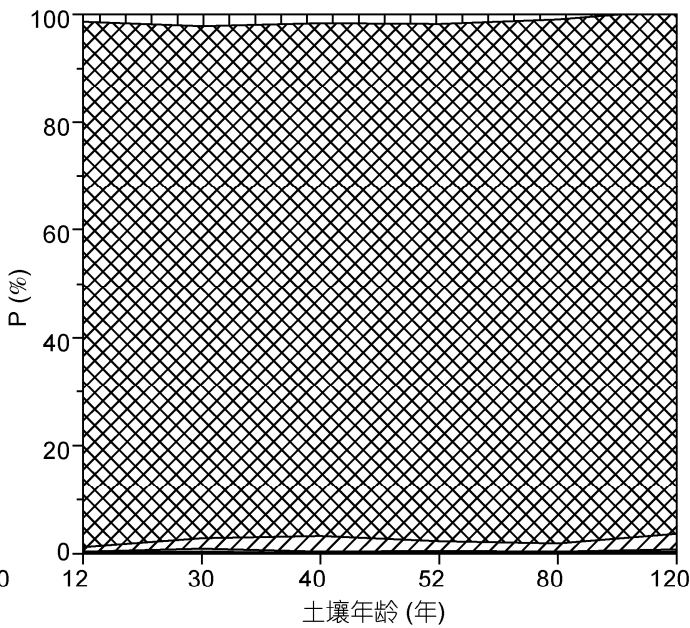

图 $3 \mathrm{C}$ 层土壤中连续提取磷形态组成 
期的 A 层进行比较. DHCl-P 从 12 年处的 $86.1 \%$ 下降 到 52 年处的 $52.7 \%$, 再下降到 120 年处的 $38.9 \%$. Resin-P 则从 12 年处的 $2.1 \%$ 逐渐增加到 80 年处的 $13.0 \%, 120$ 年处略有下降，但仍达到 $7.5 \%$. $\mathrm{NaHCO}_{3}-\mathrm{P}$ 含量的变化与 Resin-P 有相同的趋势. $\mathrm{NaOH}-\mathrm{P}$ 含量的增加可以分为 3 个阶段, 30、40 年处 $\mathrm{NaOH}-\mathrm{P}$ 含量几乎是开始阶段的两倍, 而52 年处又达 到前一阶段两倍, 80,120 年处再次翻番, 在 120 年处 $\mathrm{NaOH}-\mathrm{P}$ 占到总磷的 28.1\%(图 4(a)和(b)).

A 层土壤磷随时间变化的另一特征是有机磷的 持续增加, 由 12 年处的 $8.1 \%$ 持续增加到 120 年处的 $44.0 \%$. 有机磷以 NaOH-P 的增加最为显著, 因此从 有机磷占总磷的百分比看, 其增加也可分为三个阶 段且与 NaOH-P 的增加过程吻合(图 4(c)).

3.3 XANES 方法所获得的海螺沟冰川退缩区土壤 磷形态特征

XANES 方法对 C 层的分析表明, 海螺沟冰川退 缩区土壤 $\mathrm{C}$ 层的磷几乎只有两种形态: 钙结合态和 铝结合态, 钙结合态磷占到 $2 / 3$ 左右, 对照参考物质 其主要成份为羟基磷灰石 $\left(\mathrm{Ca}_{5}\left(\mathrm{PO}_{4}\right)_{3}(\mathrm{OH})\right)$, 铝结合态 为另 $1 / 3$ 的总磷(图 5(c)和(d)).

A 层磷的形态组成同样显示了很大变化, 最重 要的特征是有机磷的增加和䥻结合态磷的减少. 有 机磷自 30 年后迅速增加, 在 80 年处土壤磷几乎全为 有机磷, 这可能与测试样品量小造成的误差有关, 但 也可能是 XANES 方法对有机磷不能有效判识造成
(见 4.1). 铝结合态磷在 40 年以后几乎消失, 与之相 反铁结合态磷自 40 年后出现, 但含量不高, 最高在 40 年处约 $199.5 \mathrm{mg} \mathrm{kg}^{-1}$, 在 80 年处铁结合态磷尽管 只有 $14.6 \mathrm{mg} \mathrm{kg}^{-1}$, 但这是除有机磷外仅有的磷的结 形态. 钲结合态的另外两种矿物: 磷酸氢钙和磷酸二 氢钻只在 30, 40, 52 年处少量出现(图 5(a)和(b)).

\section{4 讨论}

\subsection{Hedley 连续提取法与 XANES 方法对土壤磷 形态研究的比较}

所谓生物有效磷是指生物可以直接吸收利用的 磷, 其形态为正磷酸盐, 包括溶解态正磷酸盐和一部 分颗粒态的正磷酸盐(极易从颗粒物中释放出来的弱 结合态(交换态磷), 潜在的可利用的部分通常在颗粒 物中或者溶解无机磷和聚合磷酸盐. 吸附于颗粒物 中水合金属氧化物(如无定形的铁、铝)表面上的正磷 酸根一般不能为生物所直接利用, 只有在这些水合 金属氧化物发生溶解时, 这些潜在的可利用磷才被 释放出来. 有机化合物和聚合磷酸盐在矿化过程中 可分解出生物有效态磷, 但目前人们对矿化、解吸的 过程了解甚少, 需要更多地加以探索. 其他形态的磷 包括结晶态的铝和铁磷酸盐根本不能被利用, 因为 它们通常结合在矿物晶格中难以溶解出来(Abrams 和 Jarrell, 1992; Gonsiorczyk 等, 1998; Smil, 2000; Reynolds 和 Davies, 2001).

Hedley 连续提取方法所区分出的磷的形态并不
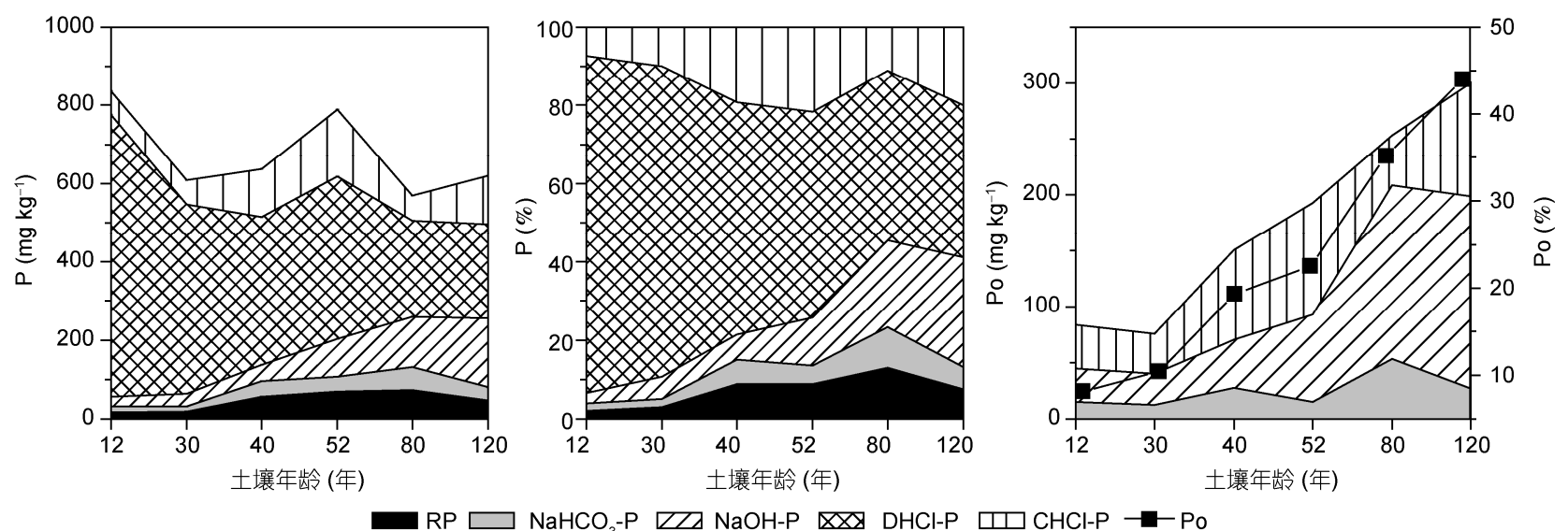

$\mathrm{NaHCO}_{3}-\mathrm{P} \square \mathrm{NaOH}-\mathrm{P} \quad \mathrm{DHCl}-\mathrm{P} \quad \square \mathrm{CHCl}-\mathrm{P} \rightarrow-\mathrm{Po}$

图 4 A 层土壤中连续提取磷形态组成

2010 

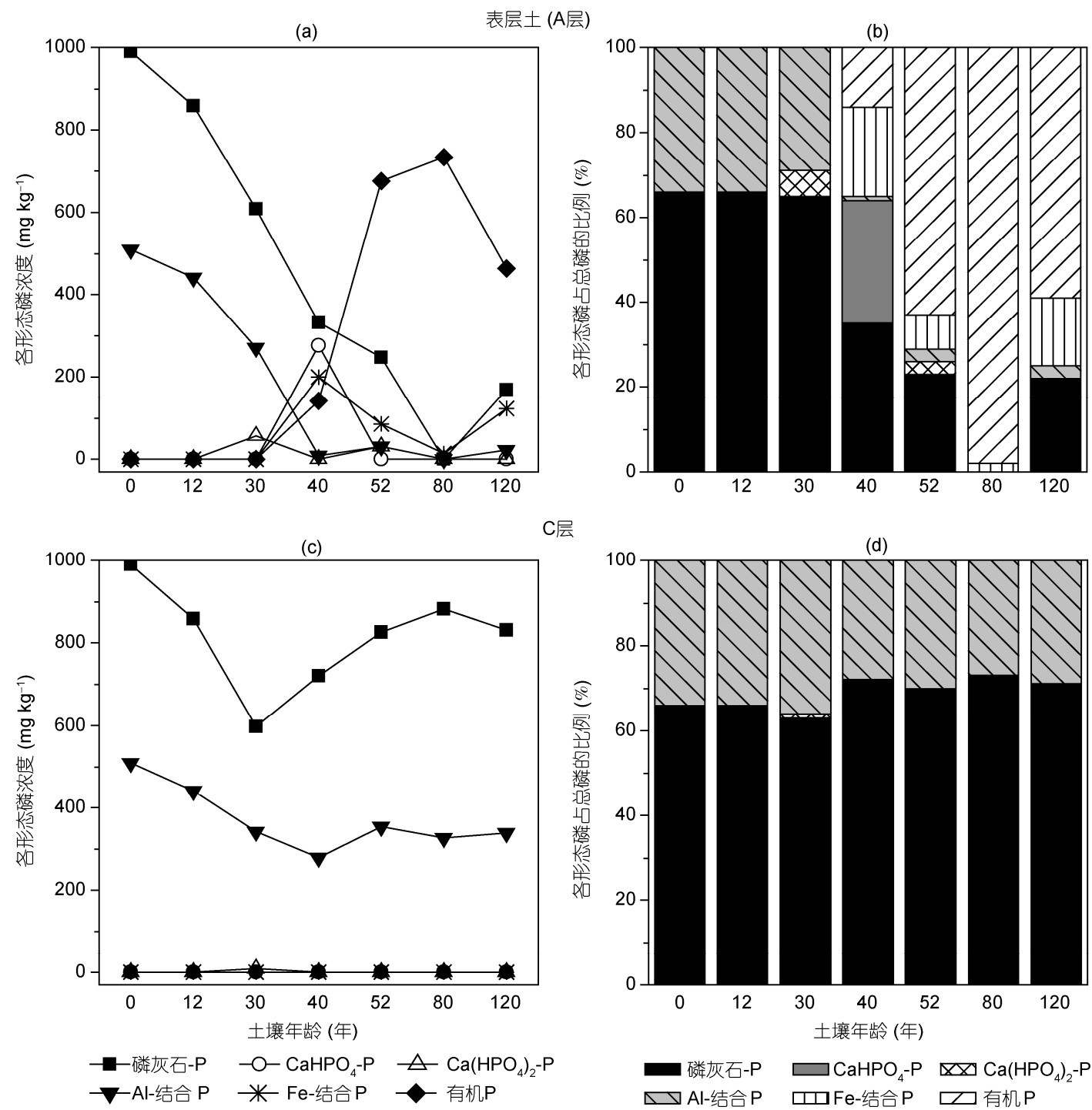

图 5 XANES 测定的 $\mathrm{A}$ 和 $\mathrm{C}$ 层土壤形态

能明确到具体的磷的化学形态或者为某种金属氧化 物或矿物所吸附(Hunger 等, 2005), 但是对溶解态正 磷酸盐形态的磷即树脂吸附的磷有比较准确的定量 估测, 也能将弱结合态的颗粒态磷通过 $\mathrm{NaHCO}_{3}$ 提取 出来. 这两种形态的磷被 Johnson 等(2003)归类为生 长季可直接为植物所利用磷, 作者且称之为即时生 物有效磷(Ready bioavailable phosphorus). 对于进一 步可转化为生物有效磷的其他形态如 $\mathrm{Al}$ 结合态的 磷、Ca 结合态磷等(Johnson 等, 2003), 以及闭蓄磷 (Walker 和 Syers, 1976)却难以区分.

XANES 方法由于是一种固相测量技术，因此不 能获得土壤中溶解态的正磷酸盐的含量(Resin-P), 但
这一方法能探知 $\mathrm{P}$ 元素周围的其他金属元素及其化 学信息(Hesterberg 等, 1999; Beauchemin 等, 2003; Lombi 等, 2006; 刘瑾等, 2011; Prietzel 等, 2013), 因 此, XANES 方法可以用以评估土壤中吸附于水合金 属氧化物的磷的含量, 作者把这部分磷称为潜在生 物可利用磷. 部分有机磷在矿化过程中也转变为生 物有效磷(Cross 和 Schlesinger, 2001; Johnson 等, 2003), 也是潜在生物可利用磷的部分. 但 XANES 所 判识的有机磷存在疑问, 这一组分到底是有机质中 所含的磷, 还是吸附于有机质的磷酸盐组分? 难以 判识. 用于确定有机磷的参考物质是肌醇六磷酸, 理 论上所判识的土壤有机磷应是存在于有机质本身的 
磷. 但 80 年处 XANES 判识有机磷占到近 $99 \%$, 而 Hedley 法测定的结果显示这一时期各提取态的有机 磷仅占总磷的 $35.2 \%$, 可见 XANES 并没能区分出吸 附于有机质或者金属有机络合物的磷.

XANES 判识的钙结合态磷与铝结合态磷有很好 的相关性 $(r=0.89)$ (表 1), 而它们与土壤形成时间显著 负相关 $(r$ 分别为 -0.96 和 -0.86$)$ (表 2$)$ 、与土壤中无定 形铁和铝也成明显的负相关关系, 显示钙结合态和 铝结合态磷随土壤发育程度增强而减少, 表明海螺 沟土壤序列中钙结合态与铝结合态磷主要代表成土 母质中的磷, 是初始矿物磷. 这与代表成土母质的 C 层的磷形态分析结果一致.

海螺沟冰川退缩迹地土壤 $\mathrm{A}$ 层两种方法所表征 的结果相关性分析显示: 钻结合态(羟基磷灰石)及铝 结合态磷与 DHCl-P 相关性较好 $(r=0.89$ 和 $r=0.83)$. Fe 结合态磷与 $\mathrm{NaHCO}_{3}-\mathrm{Pi}, \mathrm{NaOH}-\mathrm{Pi}$ 和 CHCl-Po 有良好 的相关性, 相关系数分别达到 $0.82,0.75$ 和 0.81 , 可 见弱结合态颗粒无机磷及难以分解的有机磷更倾向 于吸附于铁的水合氧化物. XANES 方法表征的有机 磷与 Resin-P 和 NaOH-Po 相关性较好. Resin-P 为无机 磷, 与 XANES 方法的有机磷显著相关, 表明土壤这 种生物有效磷越高, 生物量越大, 通过调落物分解返 回土壤的有机磷也较高. 两种方法都显示, 海螺沟冰 川退缩迹地土壤 $\mathrm{A}$ 层中一个显著特点是有机磷的显
著增加, $\mathrm{NaOH}-\mathrm{Po}$ 在 80 和 120 年含量超过 $50 \%$, 这是 XANES 有机磷与 NaOH-Po 良好相关的重要原因.

\section{2 海螺沟冰川退缩迹地土壤磷形态变化及其驱 动因素}

冰川退缩区成土作用早期, 土壤磷主要由磷灰 石组成, 这一事实在其他一些冰川前缘有类似报道 (Turner 等, 2007; Föllmi 等, 2009). 此外, 在一些花岗 岩中含有一定磷酸铝矿物如: 磷铝铁石 ( ( Fe, $\left.\mathrm{Mn}) \mathrm{AlPO}_{4}(\mathrm{OH})_{2} \cdot \mathrm{H}_{2} \mathrm{O}\right)$ 、磷铝锰石 $\left(\mathrm{MnAl}\left(\mathrm{PO}_{4}\right)(\mathrm{OH})_{2}\right.$. $\left.\mathrm{H}_{2} \mathrm{O}\right)$ 、锂磷铝石 $\left((\mathrm{Li}, \mathrm{Na}) \mathrm{Al}\left[(\mathrm{F}, \mathrm{OH})\left[\mathrm{PO}_{4}\right]\right)\right.$ 和羟锂磷铝 石 $\left(\mathrm{LiAl}\left(\mathrm{PO}_{4}\right)(\mathrm{OH})\right)$ 等 $(\mathrm{Neiva}$ 等, 2000). 海螺沟冰川堆 积物主要由花岗岩和花岗变质岩组成 ${ }^{1)}$, 其中的磷酸 铝矿物与磷灰石是 $\mathrm{C}$ 层和成土作用早期( $<12$ 年) 磷的 早期主要形态组成. 铝结合态磷矿物在成土作用过 程中较钙结合态磷更易于被释放出来, 40 年后基本不 含铝结合态磷矿物(Flicoteaux 和 Lucas, 1984). 成土 作用早期由于风化作用较弱, 磷的释放量较少, Resin-P 非常低, 尚难以满足植被发育需要.

12 年以后, 随着风化作用、成土作用增强和植被 发育, 土壤酸性增强, 磷灰石和磷酸铝矿物溶解, 铻 结合态、铝结合态磷迅速减少, 与此相反有机磷却迅 速增加, 铁结合态磷 30 年后开始出现, 与无定形铁 正相关(表 2), 但维持较低含量. 由于 XANES 法对有

表 1 XANES 表征的磷形态含量与 Hedley 连续提取各形态磷含量相关系数 a)

\begin{tabular}{|c|c|c|c|c|c|}
\hline & Ca-P1 & Ca-P3 & Al-P & $\mathrm{Fe}-\mathrm{P}$ & org-P \\
\hline Ca-P1 & 1 & & & & \\
\hline Ca-P3 & 0.04 & 1 & & & \\
\hline Al-P & 0.89 & 0.18 & 1 & & \\
\hline $\mathrm{Fe}-\mathrm{P}$ & -0.63 & -0.23 & -0.74 & 1 & \\
\hline org-P & -0.93 & -0.09 & -0.82 & 0.62 & 1 \\
\hline $\mathrm{RPi}$ & -0.83 & -0.10 & -0.83 & 0.46 & 0.93 \\
\hline NaHCO3-Pi & -0.46 & -0.05 & -0.46 & 0.82 & 0.59 \\
\hline NaHCO3-Po & -0.6 & -0.85 & -0.83 & 0.49 & 0.55 \\
\hline NaHCO3-Pt & -0.71 & -0.51 & -0.89 & 0.58 & 0.81 \\
\hline $\mathrm{NaOH}-\mathrm{Pi}$ & -0.6 & 0.07 & -0.43 & 0.75 & 0.64 \\
\hline $\mathrm{NaOH}-\mathrm{Po}$ & -0.94 & -0.27 & -0.71 & 0.58 & 0.81 \\
\hline $\mathrm{NaOH}-\mathrm{Pt}$ & -0.94 & -0.27 & -0.71 & 0.58 & 0.81 \\
\hline DHCl-Pi & 0.89 & 0.44 & 0.83 & -0.70 & -0.70 \\
\hline CHCl-Pi & 0.43 & 0.54 & 0.31 & 0.23 & -0.23 \\
\hline $\mathrm{CHCl}-\mathrm{Po}$ & -0.6 & -0.34 & -0.43 & 0.81 & 0.58 \\
\hline CHCl-Pt & -0.54 & -0.17 & -0.37 & 0.75 & 0.64 \\
\hline
\end{tabular}

a) Ca-P1 代表磷灰石磷, Ca-P3 代表磷酸二氢钻磷, 因磷酸氢钻磷只在 40 年处少量检出, 未进行相关分析

1) 何毓蓉, 张保华, 廖超林, 等. 2005. 贡嘎山东坡的土壤特征系统分类与生态环境效应. 研究报告. 中国科学院成都山地灾害与 环境研究所 
机磷区分的局限性, 如果把磷灰石磷、磷酸铝矿物磷 和铁结合态磷作为潜在可释放的磷, 海螺沟冰川退 缩区土壤序列上, 潜在可释放的磷在冰川退缩 40 年 后下降明显, 可以说表层土壤中原生矿物对土壤磷 库的贡献在 40 年后以非常有限, 甚至可以忽略 $(80$ 年处).

与潜在可释放磷变化趋势相反, 土壤中生物可 利用磷自冰川退缩 30 年后有成倍增加, 尤其是植物 可直接利用的 Resin-P, 弱结合态的 $\mathrm{NaHCO}_{3}-\mathrm{P}$ 也从 30 年后开始增加. 随着即时生物可利用磷的大量增 加, 植被开始发育, 而调落物的分解成为有机磷的主 要来源, 土壤中有机磷含量增加. 另一方面, 植被的 发育, 尤其是暗针叶林的发育, 改变了土壤性质, 如 $\mathrm{pH}$ 持续下降, 又反过来促进了含磷矿物的分解, 促 进了生物可利用磷的产生, 从此进入一个良性的 循环, 满足不同植被对磷的需求, 促使了植被演替的 发生.

由上可见, 土壤中磷的形态组成变化与土壤发 育、植被发育等密切相关. 钙铝结合态的磷与土壤发 育时间显著负相关(表 2), 与此相对应 DHCl-P 也随土 壤发育而持续下降. 随着土壤发育, 土壤中无定形铁 和铝含量逐渐增多, 对磷的吸附能力不断增强, 尤其 是无定形的铝对有机磷的吸附能力非常强, 二者之
间显著正相关 $(r=0.93)$ (表 2), 其对溶解态的 Resin-P 也有很强的吸附作用 $(r=0.83)$ (表 2). 无定形铁、铝与 土壤磷形态的显著相关关系, 充分证明土壤铁、铝羟 化物是控制土壤对磷持留能力的重要因素, 因而成 为控制磷由流域土壤向水体输出的主要因素(Kaňa 和 Kopáček, 2005).

\section{5 结论}

(1) Hedley 连续提取方法和 XANES 方法, 从不 同的方向反映了海螺沟冰川退缩区土壤磷的形态组 成变化. Hedley 连续提取法在定量表述土壤中生物即 时可利用磷方面具有优势, 而 XANES 方法由于对磷 酸盐和金属水氧化物吸附态磷有更好的分辨能力, 因而对土壤潜在生物可利用磷的评估更为准确.

(2) 海螺沟冰川退缩区土壤中铝结合态磷和钘 结合态磷主要为原始矿物磷(包括磷灰石和铝磷矿 物), 随成土作用强度增强而逐渐减少, 40 年后铝结 合态磷几乎消失. 随着土壤发育, 土壤中无定形铝增 加, 无定形铝吸附的主要是有机磷. 土壤中植物可直. 接利用磷 Resin-P 和 $\mathrm{NaHCO}_{3}-\mathrm{P}$ 从 30 年后迅速增加, 促进了植被发育, 而植被发育反过来影响土壤磷形 态组成, 表现为有机磷的持续增加和钙、铝磷的持续 减少.

表 2 海螺沟冰川退缩区土壤序列 $\mathbf{A}$ 层土壤性质与磷形态相关关系

\begin{tabular}{|c|c|c|c|c|c|c|c|c|}
\hline & Age & $\mathrm{pH}$ & Ciorg & Corg & Ntot & Ptot & Feo & Alo \\
\hline $\mathrm{Ca}-\mathrm{P} 1$ & -0.96 & 0.95 & 0.63 & -0.68 & -0.67 & 0.86 & -0.74 & -0.99 \\
\hline $\mathrm{Ca}-\mathrm{P} 3$ & -0.04 & 0.18 & 0.39 & 0.22 & 0.22 & -0.04 & -0.18 & -0.04 \\
\hline Al-P & -0.82 & 0.95 & 0.63 & -0.68 & -0.67 & 0.82 & -0.90 & -0.88 \\
\hline $\mathrm{Fe}-\mathrm{P}$ & 0.70 & -0.71 & -0.66 & 0.85 & 0.86 & -0.41 & 0.90 & 0.64 \\
\hline org-P & 0.85 & -0.86 & -0.66 & 0.70 & 0.71 & -0.63 & 0.67 & 0.93 \\
\hline $\mathrm{RPi}$ & 0.66 & -0.77 & -0.39 & 0.66 & 0.66 & -0.49 & 0.64 & 0.83 \\
\hline NaHCO3-Pi & 0.52 & -0.41 & -0.53 & 0.99 & 0.99 & 0.06 & 0.62 & 0.46 \\
\hline NaHCO3-Po & 0.49 & -0.77 & -0.65 & 0.14 & 0.14 & -0.49 & 0.72 & 0.60 \\
\hline NaHCO3-Pt & 0.54 & -0.77 & -0.65 & 0.54 & 0.54 & -0.37 & 0.75 & 0.71 \\
\hline $\mathrm{NaOH}-\mathrm{Pi}$ & 0.71 & -0.49 & -0.39 & 0.94 & 0.94 & -0.14 & 0.55 & 0.60 \\
\hline $\mathrm{NaOH}-\mathrm{Po}$ & 1 & -0.89 & -0.39 & 0.54 & 0.54 & -0.71 & 0.64 & 0.94 \\
\hline $\mathrm{NaOH}-\mathrm{Pt}$ & 1 & -0.89 & -0.39 & 0.54 & 0.54 & -0.71 & 0.64 & 0.94 \\
\hline DHCl-Pi & -0.94 & 0.94 & 0.39 & -0.49 & -0.49 & 0.77 & -0.81 & -0.89 \\
\hline CHCl-Pi & -0.37 & 0.49 & 0.13 & 0.54 & 0.54 & 0.66 & -0.06 & -0.43 \\
\hline CHCl-Po & 0.77 & -0.54 & -0.65 & 0.77 & 0.77 & -0.14 & 0.58 & 0.6 \\
\hline $\mathrm{CHCl}-\mathrm{Pt}$ & 0.66 & -0.43 & -0.65 & 0.89 & 0.89 & 0.03 & 0.49 & 0.54 \\
\hline
\end{tabular}




\section{参考文献}

李逊, 熊尚发. 1995. 贡嘎山海螺沟冰川退却迹地植被原生演替. 山地研究, 13: 109-115

刘瑾, 杨建军, 梁新强, 等. 2011. 同步辐射 X 射线吸收近边结构光谱技术在磷素固相形态研究中的应用. 应用生态学报, 22: 2757-2764

钟祥浩, 张文敬, 罗辑. 1999. 贡嘎山地区山地生态系统与环境特征. Ambio-人类环境杂志, 28: 648-654

Abrams M M, Jarrell W M. 1992. Bioavailability index for phosphorus using nonexchange resin impregnated membranes. Soil Sci Soc Am J, 56: $1532-1537$

Ajiboye B, Akinremi O O, Hu Y et al. 2008. XANES speciation of phosphorus in organically amended and fertilized vertisol and mollisol. Soil Sci Soc Am J, 72: 1256-1262

Beauchemin S, Hesterberg D, Chou J, et al. 2003. Speciation of phosphorus in phosphorusenriched agricultural soils using X-ray adsorption near-edge spectroscopy and chemical fractionation. J Environ Qual, 32: 1809-1819

Cassagne N, Remaury M, Gauquelin T, et al. 2000. Formsand profile distribution of soil phosphorus in alpine Inceptisols and Spodosols (Pyrenees, France). Geoderma, 95: 161-172

Chapin F S, Walker L R, Fastie C L, et al. 1994. Mechanisms of primary succession following deglaciation at Glacier Bay, Alaska. Ecolo Monog, 64: $149-175$

Cross A F, Schlesinge W H. 1995. A literature-review and evaluation of the Hedley fractionation-applications to the biogeochemical cycle of soil-phosphorus in natural ecosystems. Geoderma, 64: 197-214

Ducić V, Milovanović B, Durdić S. 2011. Identification of recent factors that affect the formation of the upper tree line in eastern Serbia. Arch Biol Sci, 63: 825-830

Flicoteaux R, Lucas J. 1984. Weathering of phosphate minerals. In: Nriagu J O, Moore P B, eds. Phosphate Minerals. Berlin: Springer. 292-317

Föllmi K B, Arn K, Hosein R, et al. 2009. Biogeochemical weathering in sedimentary chronosequences of the Rhône and Oberaar Glaciers (Swiss Alps): Rates and mechanisms of biotite weathering. Geoderma, 151: 270-281

Gonsiorczyk T, Casper P, Koschel R. 1998. Phosphorus-binding forms in the sediments of oligotrophic and an eutrophic hardwater lake of Baltic Lake district (Germany). Water Sci Technol, 37: 51-58

He L, Tang Y. 2008. Soil development along primary succession sequences on moraines of Hailuogou Glacier, Gongga Mountain, Sichuan, China. Catena, 72: 259-269

Hedley M J, Stewart J W B, Chauhan B S. 1982. Changes in inorganic and organic soil phosphorus fractions by cultivation practices and laboratory incubation. Soil Sci Soc Am J, 46: 970-976

Hesterberg D, Zhou W, Huchison K J, et al. 1999. XAFS study of adsorbed and mineral forms of phosphate. J Synchrotron Rad, 6: 636-638

Hunger S, Sims J T, Sparks L. 2005. How accurate is the assessment of phosphorus pools in poultry litter by sequential extraction. J Environ Qual, 34: 382-389

Johnson A H, Frizano J, Vann D R. 2003. Biogeochemical implications of labile phosphorus in forest soils determined by the Hedley fractionation procedure. Oecologia, 135: 487-499

Kar G, Hundal L S, Schoenau J, et al. 2011. DerekDirect chemical speciation of P in sequential chemical extraction residues using P K-edge X-ray absorption near-edge structure spectroscopy. Soil Sci, 176: 589-595

Kaňa J, Kopáček J. 2005. Impact of soil sorption charateristics and bedrock composition on phosphorus concentration in two Bohemian forest lakes. Water Air Soil Pollut, 173: 243-259

Kruse J, Leinweber P. 2008. Phosphorus in sequentially extracted fen peat soils: A K-edge X-ray absorption near edge structure (XANES) spectroscopy study. J Plant Nutr Soil Sci, 171: 613-620

Li Z X, He Y Q, Yang X M, et al. 2010. Changes of the Hailuogou glacier, Mt. Gongga, China, against the background of climate change during the Holocene. Quatern Int, 218: 166-175

Lombi E, Scheckel K G, Armstrong R D, et al. 2006. Speciation and distribution of phosphorus in a fertilized soil: A synchrotron-based investigation. Soil Sci Soc Am J, 70: 2038-2048

Neiva A M R, Silva M M V G, Antunes I M H R, et al. 2000. Phosphate minerals of some granitic rocks and associated quartz veins from Northern and Central Portugal. J Czech Geol Soc, 46: 35-44

Peak D, Sims J T, Sparks S. 2002. Soild-state speciation of natural and alum-amended poultry litter using XANES spectroscopy. Environ Sci Technol, 36: 4253-4261

Prietzel J, Dümig A, Wu Y H, et al. 2013. Phosphorus K-edge XANES spectroscopy reveals rapid changes of P speciation in the topsoil of two glacier foreland chronosequences. Geochim Cosmochim Acta, 108: 154-171

Ravel B, Newville M. 2005. ATHENA, ARTEMIS, HEPHAESTUS: Data analysis for X-ray absorption spectroscopy using IFEFFIT. J 
Synchrotron Rad, 12: 537-541

Reynolds C S, Davies P S. 2001. Sources and bioavailability of phosphorus fractions in freshwater: A British perspective. Biological Rev Cambridge Phil Soc, 76: 27-64

Sala O E, Chapin F S, Armesto J J, et al. 2000. Global biodiversity scenarios for the year 2100. Science, 287: 1770-1774

Smil V. 2000. Phosphorus in the environment: Natural flows and human interferences. Annu Rev Energy Environ, 25: 53-88

Tiessen H, Moir J. 1993. Characterization of available P by sequential extraction. In: Carter M R, ed. Soil Sampling and Methods of Analysis. Lewis Publishers. 75-86

Turner B L, Condron L M, Richardson S J, et al. 2007. Soil organic phosphorus transformations during pedogenesis. Ecosystems, 10: 1166-1181

Vitousek P M, Poder S, Houlton R Z, et al. 2010. Terrestrial phosphorus limitation: Mechanism, implication, and nitrogen-phosphorus interactions. Ecol Appl, 20: 5-15

Walker T W, Syers J K. 1976. The fate of phosphorus during pedogenesis. Geoderma, 15: 1-19

Wu Y H, Zhou J, Yu D, et al. 2013. Phophorus biogeochemical cycle research in mountainous ecosystem. J Mount Sci, 10: 43-53

Zhou J, Wu Y H, Prietzel J, et al. 2013. Changes of soil phosphorus stocks and speciation along a 120-yr soil chronosequence in the Hailuogou Glacier retreat area (Gongga Mountain, SW China). Geoderma, 195-196: 251-259 